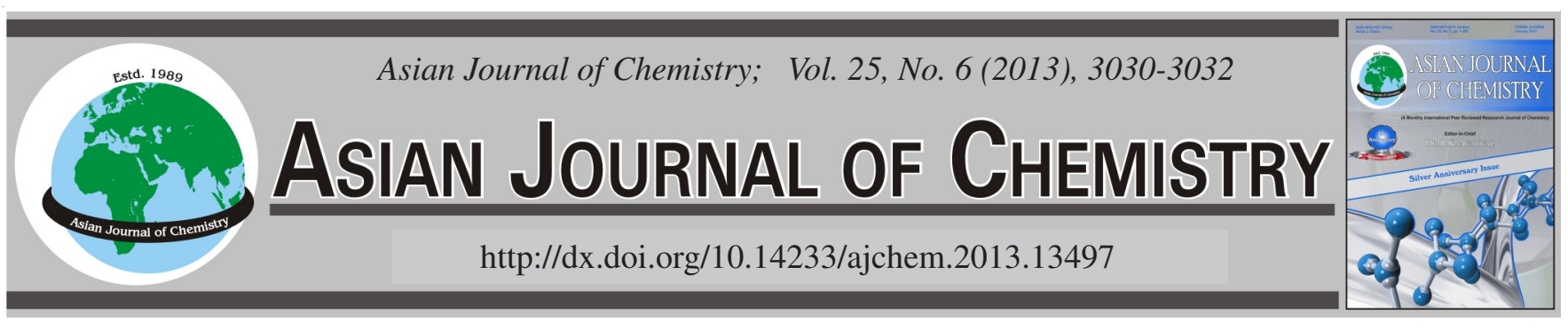

\title{
Study on the Morphologies of Poly(ethylene glycol)-block-poly( $\gamma$-benzyl L-glutamate) -graft-poly(ethylene glycol) Aggregates Deposited from the Dilute Solution
}

\author{
Guo-Quan Zhu, Fa-Gang Wang ${ }^{*}$, Guo-Chang Li, Chang-Hong Su and Yu-Ying Liu
}

School of Materials Science and Engineering, Shandong University of Technology, Zibo 255049, P.R. China

*Corresponding author: E-mail: fagangwang@126.com

(Received: 23 January 2012;

Accepted: 10 December 2012)

AJC-12512

Poly(ethylene glycol)-block-poly-( $\gamma$-benzyl L-glutamate)-graft-poly(ethylene glycol) (PEG-block-PBLG-graft-PEG) copolymer was synthe
-sized by the ester exchange reaction of PBLG-block-PEG copolymer with mPEG. The surface morphologies of PEG-block-PBLG-graft-
PEG copolymer aggregates from the dilute solution were studied by scanning electron microscopy. The effects of the solution concentration,
the precipitation temperature and various solvent system on the surface morphologies of the polypeptide block-graft copolymer aggregates
from the dilute solution were investigated.
Key Words: PEG- $\boldsymbol{b}$-PBLG-g-PEG, Aggregate, Morphology, Dilute solution. ᄂ - - - - - - - - - - - - - - - - - - - - - - - - - - -

\section{INTRODUCTION}

Polypeptides and their copolymers with excellent biocompatible and biodegradable properties have received lots of attention for their potential applications ${ }^{1-7}$. As known, the synthesized polypeptides and their copolymers have been studied widely in the fields of functional biomaterials, protein simulation, polymer carriers for protein conjugates, macromolecular conformational research, catalysis and artificial skin substrates, etc. ${ }^{8-12}$.

In order to understand the properties of polypeptides, it is important to characterize their morphologies and structures and to elucidate the relationship between their properties and the morphologies and structures ${ }^{13-15}$. Geil et al. ${ }^{14,15}$ have reported the surface morphologies of poly $(\gamma$-benzyl L-glutamate) homopolymer aggregates deposited from dilute solution. However, to our bes of knowledge, no experimental work has been done on the studies of the surface morphologies of PEG- $b$ PBLG- $g$-PEG copolymer aggregates deposited from dilute solution so far. Owing to different molecular structure of polypeptide block-graft copolymer and polypeptide homopolymer, it is still interesting to study the aggregates morphologies of PEG-block-PBLG- raft-PEG copolymer from dilute solution. In the present work, PEG-block-PBLG-graft-PEG copolymer was synthesized. SEM technique was used to study the aggregates morphologies of PEG- $b$-PBLG- $g$-PEG from the dilute solution. The effects of the solution concentration, the precipitation temperature and various solvent system on the surface morphologies of the polypeptide block-graft copolymer aggregates from the dilute solution were investigated.

\section{EXPERIMENTAL}

Amine-terminated $\alpha$-methoxy- $\omega$-amino poly(ethylene glycol) (AT-PEG, $\mathrm{M}_{\mathrm{w}}=10000$ ) and poly(ethylene glycol methyl ether) (mPEG, $M_{w}=350$ ) were purchased from Sigma Inc. (USA) and used without further purification. Hexane, tetrahydrofuran (THF) and 1,4-dioxane are of analytical grade and dried with sodium to remove water before use. All other solvents are of analytical grade and used without further purification.

Preparation of polypeptide copolymer and its solution: PBLG-block-PEG copolymer was prepared by a standard $N$ carboxyl- $\gamma$-benzyl- $L$-glutamate anhydride (NCA) method ${ }^{7}$. Molecular weight of the PBLG-block-PEG copolymer was estimated by NMR method ${ }^{2}$. The molecular weight of PBLGblock-PEG used in the study was about 90000 . PEG-blockPBLG-graft-PEG copolymer was obtained by the ester exchange reaction of PBLG-block-PEG with mPEG $\left(\mathrm{M}_{\mathrm{w}}=\right.$ 350) in 1,2-dichloroethane with $p$-toluenesulfonic acid as a catalyst according to the described $\operatorname{method}^{7}$. The grafting percentage of PEG-block-PBLG-graft-PEG was $10.5 \%$ calculated according to the document ${ }^{2}$. PEG-block-PBLGgraft-PEG copolymer solution was prepared according to the previous documents ${ }^{14,15}$. Fig. 1 shows the schematic representation of PEG-block-PBLG-graft-PEG copolymer structure.

Test method: Aggregates morphologies of polypeptide block-graft copolymer were observed by a scanning electron microscope (SEM) (Sirin 200, FEI, Holland). Testing specimens were obtained by depositing drops of precipitate suspension onto clean glass plates and drying them at room temperature ${ }^{14,15}$. 
Gold was sprayed on samples in vacuum. Acceleration voltage was $10 \mathrm{kV}$.

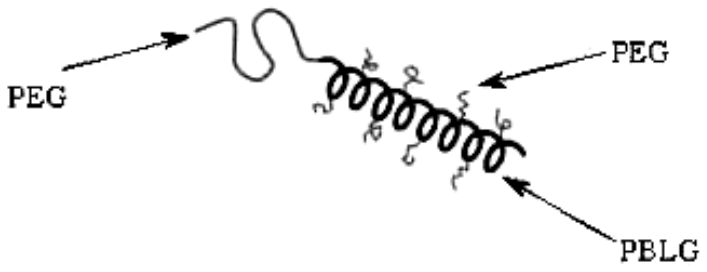

Fig. 1. Schematic representation of PEG-block-PBLG-graft-PEG copolymer structure

\section{RESULTS AND DISCUSSION}

Effects of solution concentration on the surface morphologies of PEG-block-PBLG-graft-PEG copolymer aggregates from the dilute solution: Fig. 2 shows the aggregates morphologies of PEG-block-PBLG-graft-PEG copolymer in dilute mesitylene/xylene solution with different solution concentrations. As seen from Fig. 2, with the increase of the solution concentration, the aggregates morphologies of polypeptide block-graft copolymer in dilute solution changed from dispersed spheres to tightly congregating spheres, indicating that the increase of the solution concentration changed the surface morphologies of polypeptide block-graft copolymer aggregates from dilute solution ${ }^{14,15}$. This phenomenon also proves that the solution concentration is a factor of influencing the aggregates morphologies of polypeptide block-graft copolymer in dilute solution.
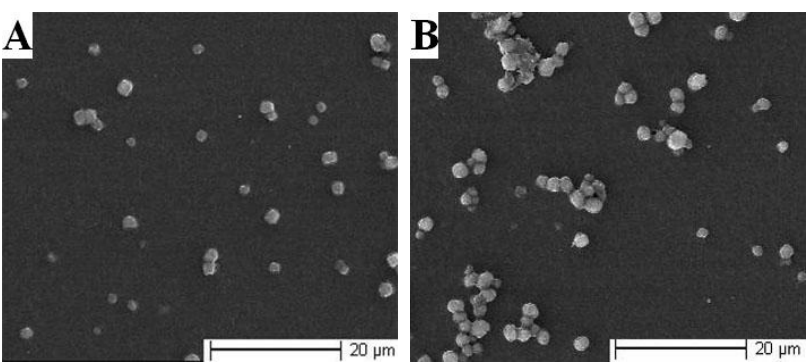

Fig. 2. SEM photographs of PEG- $b$-PBLG- $g$-PEG copolymer aggregates deposited at $40^{\circ} \mathrm{C}$ from mesitylene/xylene (vol ratio: $1: 1$ ) solutions: (a) $2 \times 10^{-4} \mathrm{~g} / \mathrm{mL}$ solution and (b) $9 \times 10^{-4} \mathrm{~g} / \mathrm{mL}$ solution, where the precipitation time is $72 \mathrm{~h}$

Effects of precipitation temperature on aggregates morphologies of PEG-block-PBLG-graft-PEG copolymer from dilute solution: Fig. 3 presents the aggregates morphologies of PEG-block-PBLG-graft-PEG copolymer from dilute toluene solution at various precipitation temperatures. As is shown in Fig. 3, the polypeptide block-graft copolymer aggregated to form fibrillar strands in dilute solution ${ }^{14,15}$. With the increase of the precipitation temperature, the fibrillar strands became thinner, shorter and having a lower aggregating density. This phenomenon indicates that the precipitation temperature is also a factor of influencing the surface morphologies of polypeptide block-graft copolymer aggregates in dilute solution.

Effects of solvent/nonsolvent mixed system on aggregates morphologies of PEG-block-PBLG-graft-PEG copolymer in dilute solution: Fig. 4 indicates the aggregates morphologies of PEG-block-PBLG-graft-PEG copolymer in dilute mesitylene/hexane solution with different hexane (as nonsolvent) volume contents. As it can be seen from Fig. 4, the surface morphologies of polypeptide block-graft copolymer aggregated to form spheres in dilute mesitylene/hexane solution. With the increase of the hexane volume content in the mixed system, the shape of the spheres became more irregular and the aggregating density increased. This phenomenon shows that the introduction of the nonsolvent into the mixed system changed the surface morphologies of the polypeptide block-graft copolymer aggregates from the dilute solution.
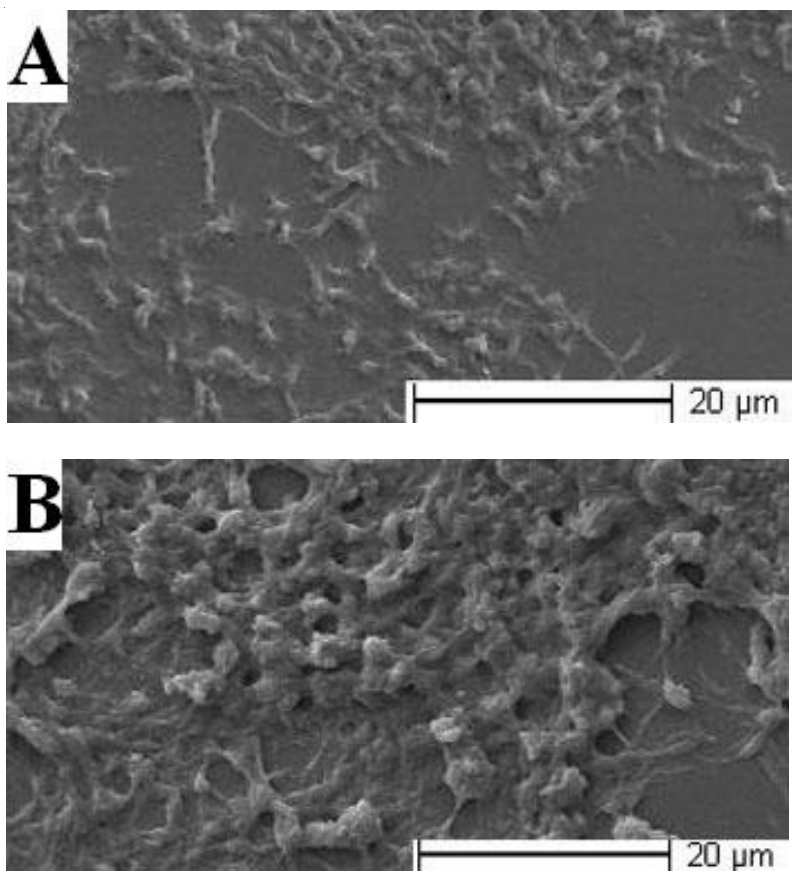

Fig. 3. SEM photographs of PEG- $b$-PBLG- $g$-PEG copolymer aggregates deposited from $7 \times 10^{-4} \mathrm{~g} / \mathrm{mL}$ various solutions: (a) toluene solution at $80{ }^{\circ} \mathrm{C}$ and (b) toluene solution at $20^{\circ} \mathrm{C}$, where the precipitation time is $72 \mathrm{~h}$
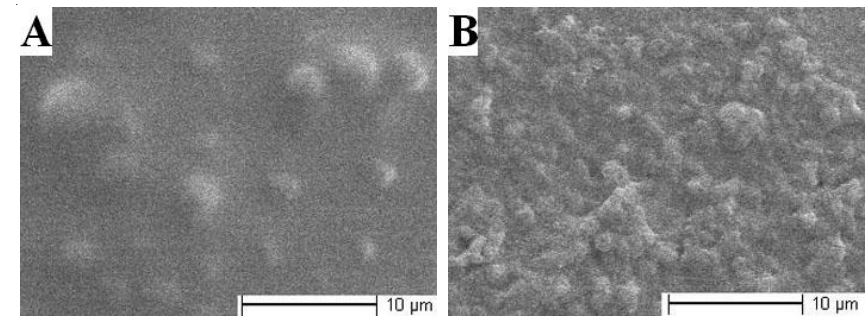

Fig. 4. SEM photographs of PEG- $b$-PBLG- $g$-PEG copolymer aggregates deposited at $30{ }^{\circ} \mathrm{C}$ from $6 \times 10^{-4} \mathrm{~g} / \mathrm{mL}$ various solutions: (a) mesitylene/hexane (vol ratio: 49: 1) and (b) mesitylene/hexane (vol ratio: $9: 1$ ), where the precipitation time is $72 \mathrm{~h}$

\section{Conclusion}

PEG-block-PBLG-graft-PEG copolymer was synthesized. The aggregates morphologies of PEG-block-PBLG- raft-PEG copolymer deposited from dilute solution were investigated by SEM technique. Experimental results demonstrated that the solution concentration, the precipitation temperature and various solvent system could exert marked effects on the aggregates morphologies of the polypeptide block-graft copolymer from dilute solution. 


\section{ACKNOWLEDGEMENTS}

This work is supported by the Natural Science Foundation of Shandong Province (No. ZR2011EMM009).

\section{REFERENCES}

1. C.S. Cho, J.B. Cheon, Y.I. Jeong, I.S. Kim, S.H. Kim and T. Akaike, Macromol. Rapid. Commun., 18, 361 (1997).

2. T. Li, J.P. Lin, T. Chen and S.N. Zhang, Polymer, 47, 4485 (2006).

3. C.S. Cho, J.W. Nah, Y.I. Jeong, J.B. Cheon, S. Asayama, H. Ise and T. Akaike, Polymer, 40, 6769 (1999).

4. G.Q. Zhu, Chem. Pap., 63, 683 (2009).

5. G.Q. Zhu, Chem. Pap., 64, 34 (2010).

6. G.Q. Zhu, L. Feng and S.N. Zhang, J. Macromol. Sci. A, 46, 694 (2009).
7. G.Q. Zhu, J. Macromol. Sci. A, 46, 892 (2009).

8. X.F. Zhong, S.K. Varshney and A. Eisenberg, Macromolecules, 25, 7160 (1992).

9. M. Moffitt and A. Eisenberg, Macromolecules, 30, 4363 (1997).

10. Z.S. Gao, A. Desjardins and A. Eisenberg, Macromolecules, 25, 1300 (1992).

11. K. Jokei, M. Oka, T. Hayashi and Y. Miyachi, Eur. Polym. J., 35, 945 (1999).

12. Y. Miyachi, K. Jokei, M. Oka and T. Hayashi, Eur. Polym. J., 35, 767 (1999).

13. J. Sun, X.S. Chen, J.S. Guo, Q. Shi, Z.G. Xie and X.B. Jing, Polymer, 50, 455 (2009).

14. F. Rybnikar and P.H. Geil, Biopolymers, 11, 271 (1972).

15. J.J.B.P. Blais and P.H. Geil, J. Ultrastruct. Res., 22, 303 (1968). 